\title{
El nuevo papel del profesor universitario de lenguas extranjeras en el proceso de convergencia europea y su relación con la interacción, la tutoría y el aprendizaje autónomo
}

\author{
María MarTínez LiRola \\ Departamento de Filología Inglesa \\ Universidad de Alicante
}

Recibido: 8 septiembre 2006 / Version aceptada: 10 octubre 2006

ISSN: $1697-7467$

\begin{abstract}
Resumen: Este artículo enfatizará dos cuestiones fundamentales en la práctica de la enseñanza actual de idiomas que se han de potenciar para que los alumnos adquieran protagonismo en el proceso de enseñanza-aprendizaje: el nuevo papel del profesor de lenguas extranjeras como motivador y facilitador propuesto por el proceso de convergencia europea y la importancia de la interacción en la clase. El nuevo papel del docente de idiomas será conectado con la importancia del aprendizaje autónomo y las tutorías dentro del marco europeo de educación.

Este trabajo revisará los principales papeles que el profesor de idiomas puede asumir en su práctica docente y pondrá de manifiesto que el papel del docente de idiomas determina el tipo de discurso que éste emplea en el aula. En consecuencia, al promoverse un cambio en dicho papel con el proceso de convergencia europea, también se promueve un cambio en el tipo de enseñanza, en la que la interacción en el aula ha de ser fundamental.

Palabras clave: Espacio Europeo de Educación Superior (EEES), papeles del profesor universitario de idiomas, enseñanza de lenguas extranjeras, interacción, proceso de enseñanza-aprendizaje, aprendizaje autónomo, tutoría.
\end{abstract}

\footnotetext{
Abstract: "This paper intends to emphasize two important issues in current language teaching practice that must be highlighted so that students become the protagonists in the teaching-learning process: the new role of the teacher of foreign languages as motivator and facilitator proposed by the process of European convergence, and the importance of interaction in the classroom. The new language teacher's role will be connected with the importance of autonomous learning and tutorials within the European educational framework.

This study will revise the main roles that language teachers can have in their teaching practice and will also highlight that the language teacher's role determines the type of discourse he/she uses in the classroom. Consequently, since the process of European convergence promotes a change in the teacher's role, it also promotes a change in the teaching practice, in which interaction in the classroom must be crucial.

Key words: European Space of Higher Education, foreign languages' lectures roles, foreign language teaching, interaction, teaching-learning process, autonomous learning, tutorials.
} 


\section{InTROduCCIÓN: una APROXIMACIÓN AL PROCESO DE CONVERGENCIA EUROPEA}

El proceso de construcción del Espacio Europeo de Educación Superior (EEES) se inicia con la Declaración de la Sorbona de 1998 y se consolida con la Declaración de Bolonia de 1999, en la que se impulsa la reorganización del sistema universitario para adaptarse a un modelo común europeo. El EEES tiene por objeto la adopción de un sistema de titulaciones flexible a nivel europeo que facilite la movilidad de los estudiantes, promueva las oportunidades de trabajo y genere una mayor competitividad internacional. Este nuevo sistema implica una profunda reestructuración de la docencia universitaria en lo concerniente al diseño curricular, a las estrategias de aprendizaje y a los modelos de evaluación.

El proceso de convergencia del EEES deberá verse completado en 2010 y se articula en torno al Sistema Europeo de Transferencia de Créditos (ECTS), el cual se halla actualmente implantado en un buen número de países de la Unión Europea y constituye el punto de referencia básico para lograr la transparencia y la armonización de las enseñanzas.

El proceso de convergencia europea lleva consigo una serie de cambios en la misión de la universidad: ha de ser una universidad formativa y educadora capaz de crear profesionales competentes, cultos, responsables, reflexivos, críticos y con capacidad de adaptación. Tal y como consta en el Real Decreto 56/2005, de 21 de enero de 2005: "La contribución del sistema universitario español a la conformación de los Espacios Europeos de Educación Superior y de Investigación y su plena integración en ellos constituye uno de los principales elementos, en la sociedad del conocimiento, para la consecución del objetivo trazado en las Cumbres de Lisboa y Barcelona para lograr que los sistemas educativos europeos se conviertan en una referencia de calidad mundial para el año 2010."

Con el EEES se produce un cambio de la universidad del enseñar a la universidad del aprender; de igual modo hay un cambio de énfasis del suministro de información (input) a los resultados del aprendizaje (output). Los cambios en la docencia en el nuevo sistema están propuestos con el fin de mejorar la calidad de la enseñanza. En lo que respecta a la enseñanza de idiomas, se pretende que el profesor pueda facilitar el proceso de aprendizaje de los alumnos haciendo que éstos sean conscientes de las diferentes estrategias de aprendizaje que pueden seleccionar tanto durante su aprendizaje de la lengua extranjera como durante el uso de la misma. Estamos de acuerdo con Cohen (1998: 65) en que "[...] the most efficient way for learner awareness to be heightened is that by having teachers provide strategies-based instruction to students as part of the foreign language curriculum." De este modo el alumno se beneficiará del uso efectivo del aprendizaje de la lengua y del uso de la lengua, factor que se relaciona de un modo directo con los papeles que los docentes de idiomas deciden asumir en la clase.

Además, el proceso de convergencia exige una serie de cambios globales, es decir, se han de armonizar los sistemas universitarios europeos mediante los siguientes elementos:

- Sistema homogéneo de titulaciones (Grado y Postgrado).

- Una misma valoración de la carga lectiva.

- Estructura de cursos y contenidos entendibles por todos los Estados.

Para ello, se han de llevar a cabo unos cambios en la docencia con el fin de mejorar la calidad de la enseñanza. Los principales propósitos con los que surge este cambio son los siguientes: 
María Martínez Lirola $\quad$ El nuevo papel del profesor universitario de lenguas extranjeras ...

- Docencia centrada en el alumno (aprendizaje autónomo, técnicas estudio) .

- Diferente papel del profesor (como gestor del proceso de aprendizaje).

- Definición más clara de los objetivos (competencias).

- Nueva organización de las actividades (shift from input to output).

- Cambios en la organización del aprendizaje (modularidad).

- Preparar al estudiante para el aprendizaje autónomo, pero acompañado.

- Dar más importancia al manejo de herramientas de aprendizaje que a la acumulación de conocimientos.

- Equilibrar la relación exigencias-apoyo para el aprendizaje.

- Docencia ajustada a los parámetros curriculares: continuidad.

Uno de los pilares fundamentales del nuevo modelo educativo es el aprendizaje por competencias. El Common European Framework dedica el capítulo quinto a las competencias del alumno, de modo que éste adquiera protagonismo en las situaciones comunicativas:

In order to carry out the tasks and activities required to deal with the communicative situations in which they are involved, users and learners draw upon a number of competences developed in the course of the previous experience. In return, participation in communicative events (including, of course, those events specifically designed to promote language learning) results in the further development of the learner's competences, for both immediate and longterm use. (Council of Europe, Common European Framework, 2001:101).

La convergencia europea supone un proceso de aprendizaje-enseñanza de competencias, entendidas como actitudes o capacidades, que preparan al alumno para responder a las demandas de su entorno profesional y laboral y que permiten concretar los resultados del aprendizaje. La competencia puede definirse como: "Un saber hacer complejo resultado de la integración, movilización y adecuación de capacidades, habilidades y conocimientos utilizados eficazmente en situaciones que tengan un carácter común" (Lasnier, 2000).

La formación de competencias viene determinada por los siguientes factores:

- Sociedad del conocimiento

- Cultura de la calidad

- Desarrollo de un nuevo paradigma, que lleva consigo los siguientes cambios:

- Del enseñar al aprender

- Énfasis en los resultados del proceso de enseñanza-aprendizaje.

- Modelos teóricos sobre el aprendizaje

- Principios de la formación de competencias: cognitivismo, constructivismo y socioconstructivismo.

- Modelo integrado de aprendizaje, enseñanza y evaluación

- Nuevas tareas para profesor y alumno.

Hay distintos tipos de competencias, siguiendo la clasificación que aparece en el Proyecto Tuning (González y Wagenaar, 2003: 79 y ss.): las competencias pueden ser generales y se caracterizan por ser las habilidades necesarias para el empleo y la vida como ciudadano. Éstas son importantes para todos los alumnos, con independencia de cual sea la carrera que se curse. Las competencias también pueden ser específicas, es decir, las habilidades propias o vinculadas a una titulación que le dan identidad y consistencia social y profesional al perfil formativo. 
En la enseñanza de idiomas, las principales competencias generales y específicas son las siguientes (cf. Aleson et al., 2005: 131):

1) Competencias generales

- Conocimientos

- Destrezas

- Competencia existencial

- Capacidad de aprender

2) Competencias comunicativas

- Competencias lingüísticas

- Competencias sociolingüísticas

- Competencias pragmáticas

En este artículo vamos a prestar atención a dos aspectos fundamentales que se han de potenciar en el nuevo modelo de enseñanza para que el alumno adquiera protagonismo y el aprendizaje tenga lugar por medio de competencias y no con objetivos, como se ha hecho tradicionalmente: por un lado, un cambio en el papel del profesor y su relación con el aprendizaje autónomo y las tutorías como factores indispensables para que el aprendizaje efectivo tenga lugar y, por otro lado, el fomento de la interacción en el aula. Esto implica un profundo cambio en la enseñanza que pasa de estar centrada en la lección magistral en la que el profesor tiene todo el protagonismo, a ser una enseñanza interactiva, en la que el alumno participa activamente, hecho que implica un cambio en el papel de profesor, como veremos en el apartado siguiente.

\section{LoS PAPELES PRINCIPALES DEL PROFESOR UNIVERSITARIO DE IDIOMAS}

Si la clase es el lugar en que queremos que el alumno aprenda y experimente la lengua extranjera, es importante establecer un clima en que los alumnos no estén cohibidos, agresivos o con miedo. Deben sentirse motivados por el contexto de la clase y ver al profesor como ayudante y facilitador (Stern, 1992:320). Para conseguir esto es fundamental que el profesor de idiomas asuma el rol o roles necesarios en la enseñanza, que estará muy relacionado con lo que pretenda que el alumno alcance mientras aprende dentro y fuera del aula.

Con la creación del Espacio Europeo de Educación Superior (EEES), se produce un cambio en el papel del profesor pues de ser el protagonista de la enseñanza al ser la persona que estructura el proceso de aprendizaje, el supervisor y director de trabajos, pasa a ser "un acompañante en el proceso de aprender, que ayuda al que estudia a alcanzar ciertas competencias" (González y Wagenaar, 2003:74). El profesor de idiomas tiene que asumir nuevos roles con la convergencia europea: motivador, consejero, orientador, facilitador, observador, planificador, tutor, supervisor, etc. Algunos de estos roles o papeles se desempeñarán de manera virtual mediante el uso de las nuevas tecnologías.

Los roles tradicionales del profesor consisten en ser fuente de conocimientos y en controlar cada aspecto del proceso de enseñanza-aprendizaje. Siguiendo a Harmer (2001:57 y ss.) los papeles del profesor de idiomas serían los siguientes: facilitador, organizador, asesor, tutor y observador de cómo se desarrolla el proceso de aprendizaje. De manera similar, McDonough y Shaw (1993:295), ponen de manifiesto los siguientes roles del profesor que enseña lenguas 
extranjeras o segundas lenguas (a) controlador de todo lo que ocurre en clase; (b) organizador de las actividades de clase; (c) evaluador de los conocimientos adquiridos; (d) participantes de actividades de debate o role-plays; y (e) fuente de conocimientos y consejo. Legutke y Thomas (1991) señalan que el profesor desempeña los siguientes papeles: como coordinador y facilitador del aprendizaje, como manager y organizador, como instructor y como investigador.

Alcaraz Varó y Moody (1983:14) distinguen de forma preliminar, entre influencia directa e influencia indirecta del profesor de idiomas. En la primera ubicaríamos al profesor que acepta y reconoce el sentir de los alumnos, el que anima y aprueba lo que hace el alumno, el que usa las ideas del alumno, y el profesor que pregunta. En la segunda ubicaríamos al profesor que explica u orienta, al que da instrucciones y al que critica o justifica su autoridad.

Perrenoud (1999) señala que entre las nuevas competencias docentes hay que destacar las siguientes: organizar y dinamizar situaciones de aprendizaje, gestionar los progresos en el aprendizaje, implicar a los estudiantes en su aprendizaje y esfuerzo, trabajar en equipo y formar a los estudiantes en el trabajo en grupo, afrontar los deberes y dilemas éticos de la profesión y gestionar su propia formación continua.

Carriscondo Esquivel (2002: 57) pone de manifiesto que el profesor ha de cumplir con dos funciones específicas que a nuestro juicio se han de tener muy presentes en el nuevo modelo de enseñanza propuesto por el proceso de convergencia europea, las cuales se llevan a cabo en dos momentos diferentes: (1) Planear y diseñar las experiencias y actividades necesarias para la adquisición de los aprendizajes previstos, así como definir los espacios y recursos adecuados para su logro; ${ }^{1}$ y (2) facilitar, guiar, motivar y ayudar a los alumnos durante su proceso de aprendizaje, así como guiar permanentemente el curso hacia los objetivos propuestos.

Basándonos en las dos funciones específicas del profesor a las que nos acabamos de referir, en nuestra opinión, los principales papeles que un profesor universitario de idiomas ha de asumir son los siguientes:

No hay duda de que el profesor es un orientador, guía o facilitador del aprendizaje ya que el profesor es un puente entre la clase y la sociedad y la cultura a la que representa la lengua extranjera. De este modo, muchos de los estereotipos que normalmente van unidos a los hablantes de una lengua determinada pueden desaparecer o al menos transformarse.

El papel del profesor como manager y organizador es determinante a la hora de preparar las diferentes actividades que harán que el proceso de aprendizaje sea satisfactorio por medio de dichas actividades y de la metodología adecuada. El profesor ha de controlar y organizar las interacciones en el aula, es decir, es fundamental que el profesor explique con claridad lo que los alumnos han de hacer y dé las instrucciones de una manera precisa.

El docente de idiomas también ha de instruir e informar, es decir, ha de transmitir conocimientos y enseñar a los alumnos cómo aprender por medio de la experiencia. De este modo, el profesor ha de intentar que los alumnos encuentren una relación entre sus propias experiencias e intereses con el contenido de la clase. Tal y como consta en Hall (2000:294): "Motivating learners to make connections between their own and other's background knowledge and experiences and to share the connections with each other promotes their extended engagement

\footnotetext{
${ }^{1}$ Esta actividad del profesor es previa al desarrollo del curso.
} 
in the interactions. This, in turn, facilitates their development as both learners and users of the new language."

Por otro lado, el profesor ha de investigar constantemente de modo que pueda mejorar su práctica docente, contrastar lo que ocurre en sus clases con otras experiencias y experimentar en el aula con el fin de facilitar el aprendizaje de los alumnos. En opinión de Legutke y Thomas (1991:303), el profesor como investigador tiene la responsabilidad de "[...] engage actively in researching what is happening in the classroom with the view to understanding its processes more clearly and to bringing about improvements".

El profesor de idiomas tiene también un papel de evaluador de las contribuciones y el trabajo del alumno debido a que juzga si las tareas que el alumno desarrolla son correctas y relevantes. Además, el profesor ha de ser considerado un guía u organizador de la asignatura que imparte debido a que da las pautas de cómo ha de aprenderse esa asignatura y selecciona entre los diferentes materiales los más adecuados para que el aprendizaje sea efectivo.

En las clases el profesor es considerado un experto, cuyo papel es estructurar la materia que es objeto de estudio y controlar el aprendizaje de los alumnos, es decir, el profesor ha de ser una fuente de conocimientos sobre la asignatura y ha de conocer diferentes materiales y recursos disponibles para que el proceso de enseñanza sea efectivo. Harmer (2001:242) expone que este papel tiene dos aspectos: "One is to be aware of what is going on as an assessor - although discreetly - and the other is to be a kind of walking resource centre."

Además de ser capaz de asumir diferentes papeles en diferentes momentos del proceso de enseñanza-aprendizaje, es evidente que el profesor de una lengua extranjera debe poseer unas cualidades personales determinadas así como formación lingüística y pedagógica. Alcaraz y Moody (1983:5-6) destacan las siguientes cualidades personales: vocación para la enseñanza, personalidad abierta y dinámica, proyección humanística y voluntad permanente de puesta al día. Estamos de acuerdo con Mateo Martínez (1999:76) en la siguiente afirmación:

El profesor enseña pero también debe aprender a enseñar. Ha de aprender a predecir, como hemos visto, las necesidades docentes de sus alumnos y adaptar sus métodos al nivel intelectual de éstos. Debería regirse por los principios de cooperación y solidaridad que define Grice y saber cuando se produce la transmisión de conocimientos y cuando se interrumpe. Ha de aprender, en definitiva, a inducir el deseo de aprender del alumno. Por su lado, el alumno, estimulado por la voluntad interactiva del profesor, ha de participar activamente en el aula, tomando las riendas cuando el profesor o la actividad se lo exija. Debe cambiar ciertos hábitos acomodaticios y pasivos, convencerse de que es, en realidad, el centro del proceso y afrontar con decisión ese reto.

El contexto de la clase permite ver a alumnos y profesores como miembros del contexto social en el que se enmarca la práctica docente; además, permite entender el discurso que éstos emplean con funciones sociales y pedagógicas. Tal y como consta el Hall y Verplaetse (2000:10) por medio de las acciones comunicativas del profesor de idiomas, éste modela el desarrollo de los alumnos de los siguientes modos:

First, they make salient to the learners certain properties of the world constituted within their classrooms, providing models of what they consider appropriate communicative actions and ways to go about acquiring those actions as they do. Second, they mediate both the quantity and quality of opportunities the students will have to participate in and learn from the activities. In doing so, they make 
María Martínez Lirola $\quad$ El nuevo papel del profesor universitario de lenguas extranjeras ...

visible their own attitudes toward the activities and toward the students' involvement in them. This, in turn, shapes the degree of individual learning that will occur. Third, in and through their interactions with learners, teachers make apparent the standards against which students' performances are measured.

Potenciar los nuevos papeles del docente de idiomas propuestos por el proceso de convergencia europea -supervisor, motivador, tutor, facilitador, asesor, etc.- está muy relacionado con el protagonismo que adquiere el aprendizaje autónomo en la universidad. El aprendizaje autónomo es una forma de aprendizaje en la que el estudiante asume una parte importante de la responsabilidad de la organización de su trabajo ajustándola a su propio ritmo. Para ello han de darse una serie de condiciones que enumeramos a continuación:

- Requiere de un sistema intenso de tutoría.

- Exige mayor esfuerzo docente que las lecciones.

- Exige una preparación del alumnado en el dominio de ciertas técnicas de trabajo (incluidas las TIC).

Con este aprendizaje se consigue que el alumno sea autónomo, es decir, un alumno con las siguientes competencias:

- Capacidad de iniciativa.

- Saber configurar un plan de trabajo realista.

- Manejarse con fuentes de información (y saber contrastarlas).

- Comprender informaciones y textos. Resumirlos.

- Plantear y resolver problemas.

- Voluntad por conocer cosas nuevas y profundizar en ellas.

- Transferir, extrapolar y aplicar conocimientos a situaciones nuevas.

- Reflexionar y evaluar sobre su propio trabajo.

En el aprendizaje autónomo la tutoría juega un papel fundamental. El EEES, junto con el diseño de la enseñanza esencial y no presencial, contempla la importancia de la tutoría docente como parte esencial del trabajo curricular. En este sentido, Carrasco Embuena y Lapeña Pérez (2005: 330) señalan que:

Los procesos de orientación y tutoría son indicadores de calidad de las instituciones de educación superior, en este momento crucial de la redefinición de los procesos de formación y de ajuste de los mecanismos para facilitar la transición entre los diferentes sistemas de formación y de ocupación.

En la actualidad las tutorías son un recurso que el profesor puede utilizar para individualizar la enseñanza y ajustarla a las características esenciales de cada estudiante. Es ésta una necesidad urgente en una enseñanza masificada donde la relación y comunicación entre profesor y cada alumno es difícil siendo importante en el proceso de aprendizaje. Carrasco Embuena y Lapeña Pérez (2005: 336-337) se centran en los distintos formatos que puede 
tener una tutoría: obligatoria, voluntaria, individual o grupal. Además, ponen de manifiesto que la tutoría puede tener una serie de rasgos pues puede ser presencial, virtual o en foros virtuales.

La tutoría es un método centrado en el alumno frente a otros centrados en el profesor, como la clase magistral, o centrados en la materia como ocurre con el seminario. En las tutorías el profesor se preocupa de desarrollar las capacidades e intereses específicos de los alumnos y de ofrecer retroalimentación del trabajo realizado por cada alumno. El antecedente histórico de este método es el sistema tutorial inglés.

\section{EL PROFESOR DE IDIOMAS COMO MOTIVADOR Y FACILITADOR: LA IMPORTAN- CIA DE LA INTERACCIÓN EN EL AULA}

Tradicionalmente, la lección magistral es la técnica más utilizada para enseñar en la universidad debido a que se puede usar en clases muy numerosas pero presenta un inconveniente importante ya que el profesor monopoliza el discurso y los alumnos apenas pueden participar. En este sentido, concurrimos con Consolo (2000:105) en que: "Most of the time teachers and students rigidly observe their part in the socially defined classroom roles. Although they may engage in a spoken discourse that resembles conversational English, they do so to achieve their teaching and learning goals."

Los patrones de comunicación que se establecen en las clases de lenguas extranjeras son construidos normalmente por los profesores. En general, podemos afirmar que el discurso del aula está bastante estereotipado y tanto alumnos como profesores lo pueden predecir. Estamos de acuerdo con Alcón Soler (2001:272) al referirse a la asimetría de poder entre los interlocutores que participan en la comunicación que tiene lugar en el aula:

En la mayoría de los casos el profesor controla y dirige la interacción en el aula: decide quién participa, selecciona al interlocutor y evalúa la participación de los interlocutores en el discurso. En muchas ocasiones el profesor también modifica el input al que son expuestos los estudiantes o la estructura de la interacción con la finalidad de facilitar la comprensión y la producción de la lengua objeto de estudio.

Es decir, se esfuerza por favorecer la comunicación en el aula.

El papel del profesor de idiomas determina el tipo de discurso que éste emplea en la práctica docente, de ahí que, al promoverse con el proceso de convergencia europea un cambio en dicho papel, también se promueva un cambio en el tipo de enseñanza, en la que la enseñanza interactiva ha de adquirir protagonismo. En palabras de Martínez Lirola (2005: 2): "The teacher's role determines the type of discourse he/she uses in the classroom and the unity between the role and the discourse directs the teaching process and determines the improvements of students in the foreign language."

Con la convergencia europea el docente de idiomas adquiere un papel fundamental como facilitador y motivador. Estos dos papeles tienen especial relevancia en las clases interactivas ya que le profesor ha de encargarse de facilitar que el aula sea un lugar seguro en que los alumnos puedan discutir y practicar; además, el profesor ha de motivar a los alumnos a intervenir activamente en las discusiones y actividades propuestas, de modo que el alumno 
se convierta en el protagonista de lo que ocurre en el aula y asuma responsabilidad en su proceso de aprendizaje.

La lengua de la clase tiene un papel fundamental en la negociación de los significados, en la clarificación de las tareas que los alumnos han de realizar, en el proceso de exploración de las dificultades de los alumnos y en la evaluación de los progresos de los alumnos, tal y como afirma Christie (2000:184) "The principal resource available to teachers and students with which to achieve educational goals is language", de ahí que entendamos la lengua como "a systematised resource which people draw on differentially, depending on what they are trying to achieve in different social and cultural contexts." (De Sylva Joyce y Burns, 1999:14)

La interacción nos permite hablar de un proceso de colaboración entre estudiantes y profesores en el proceso de enseñanza-aprendizaje, es decir, es una actividad combinada. Siguiendo a Morell Moll (2004:50), usaremos el término clase interactiva para referirnos a las clases en las que se fomenta la participación de los alumnos. El término 'interacción en el aula' ("classroom interaction") se refiere a la interacción entre el profesor y los alumnos y entre los alumnos en la clase (Tsui, 2001:120). Por su parte, Ellis (1990:93) señala que la enseñanza como interacción consiste en "the process by which samples of the target language become available to the learner for interlanguage construction through classroom interaction." De este modo, entendemos que todo lo que tiene lugar en la clase contribuye a una comunicación efectiva.

A nuestro juicio, tanto los alumnos de lenguas extranjeras o de segundas lenguas como los profesores de idiomas han de entender la interacción como una estrategia para aprender la lengua, entendiendo por estrategia los pensamientos y pensamientos empleados por los alumnos y profesores con el fin de mejorar el aprendizaje y de comprender mejor cómo funciona la lengua extranjera (Cohen, 1998:68).

Tras lo expuesto en líneas anteriores, es evidente que estamos a favor de prácticas docentes que favorezcan las tareas comunicativas de modo que la participación de los alumnos sea real y fundamental en el proceso de aprendizaje. Dichas tareas han de tener las siguientes características (Vez, 2001:270):

- Representativa de procesos de comunicación en la vida real.

- Identificable como unidad de actividad en el aula.

- Dirigida intencionalmente hacia el aprendizaje de la lengua.

- Diseñada con un objetivo, estructura y secuencia del trabajo.

- Orientada a la consecución de un objetivo de manipulación de información y significados.

El discurso del profesor de idiomas tiene un papel central en el aprendizaje de una lengua extranjera pues de la calidad de ese discurso depende en cierta medida la comprensión y el aprendizaje del alumno. En este sentido, Ellis (1994:573-574) declara que la interacción en el aula "is not planned in advance, but rather is 'co-produced' with the learners. In part, it will reflect the pedagogic decisions that have been taken, but it will also evolve as part of the process of accomplishing the lesson. The interaction provides learners with opportunities to encounter input or to practise the L2."

Normalmente el papel del docente en la interacción es superior y dominante con respecto al del alumno porque los profesores controlan el contenido de la estructura de la comunicación en el aula pues se ha establecido así socialmente. A nuestro juicio, esta idea precon- 
cebida ha de modificarse para que el alumno asuma su papel de protagonista en el proceso de aprendizaje, en palabras de Morell Moll (2004:41):

[...] si el profesor decide que es el experto en la materia y su tarea es transmitir una información que se ciñe estrictamente al contenido teórico en las horas destinadas a ese curso, sin más, el poder resulta ser extremadamente desigual o asimétrico. Del mismo modo, si el contacto se limita a las horas establecidas y no se crea un ambiente receptivo, probablemente el alumno tomará un papel pasivo y la implicación afectiva será más baja. Por el contrario, se puede ceder parte del poder a los alumnos a través del lenguaje, tanto el verbal como el no verbal, para que ellos asuman un papel más activo y de este modo incrementen la implicación afectiva que, a su vez, crea un ambiente más receptivo y propicio para el aprendizaje.

La cita anterior pone de manifiesto que es importante que tenga lugar un cambio de responsabilidades en la clase de modo que el profesor de idiomas deje de controlar cada aspecto del proceso de aprendizaje y los alumnos dejen de ser totalmente dependientes del profesor. De este modo, el profesor pasa de ser un controlador o instructor a ser un facilitador del proceso de aprendizaje, es decir, su papel consiste en ayudar a los alumnos a que sean independientes y responsables de su propio proceso de aprendizaje. (Cohen, 1998:97)

Es evidente que por medio del discurso entre el profesor y el alumno se da forma $\mathrm{y}$ contenido a la lengua extranjera con el fin de que se mejore el aprendizaje del alumno, que es el protagonista del proceso de aprendizaje, tal y como señala Mateo Martínez (1999:73):

El proceso docente, en líneas generales, ha pasado de ser monocéntrico y jerarquizado a ser multicéntrico y dirigido a satisfacer los intereses de aprendizaje de los alumnos. El profesor ha visto modificado en grado sumo su papel, aunque continúa siendo el eslabón imprescindible que permite el acceso de los alumnos al conocimiento.

No hay duda de que el discurso del profesor que enseña idiomas y del alumno que los aprende tiene funciones pedagógicas y sociales en el aula pues ambos son miembros de un contexto sociolingüístico: el aula. En este sentido, Hall y Verplaetse (2000:5) declaran que:

[...] there has been quite a bit of work attempting to define the features of teacher talk considered crucial to its role in making the message comprensible. These investigations have included the examination of such features as the degree of syntactic complexity, the rate of speech, and the length of utterance. Discourse features of teacher talk such as feedback, error correction, and use of questions have also been examined for the roles they play in making talk comprehensible.

A través de la interacción, el profesor consigue afianzar el proceso de aprendizaje de los alumnos. La interacción nos permite hablar de un proceso de colaboración entre el alumno y el profesor en el proceso de enseñanza/aprendizaje, es decir, se trata de una actividad conjunta, de este modo, el profesor deja de tener una posición dominante en función del papel social que ocupa y se ocupa de organizar clases interactivas. (Martínez Lirola, 2005:8)

Las clases interactivas de enseñanza de lenguas extranjeras conceden al alumno oportunidades para desarrollar sus capacidades como futuros profesores al ser agentes activos de su propio aprendizaje. A este respecto, nos parece muy clara la afirmación de Hall (2000:294295): 
Finally, as teachers of either language or content we must understand the inextricable link between our classroom practices and student development and, more specifically, the significant role we play in creating conditions that define both the substance and direction of student development, and use this knowledge to improve on our own practices.

\section{Conclusiones}

La clase de idiomas ha de ser un auténtico acto social en el que intervengan por medio de un discurso interactivo tanto el profesor como los alumnos. El profesor es un mediador entre los alumnos y la materia que es objeto de estudio, de ahí que cuanto mayor es la interacción en el aula, mayor es el grado de aprendizaje de la lengua extranjera, es decir, los profesores con una vocación comunicativa e interactiva obtienen mejores resultados.

El discurso del profesor de idiomas es fundamental en el proceso de aprendizaje ya que cuando el discurso es claro e invita a la participación activa de los alumnos, el aprendizaje suele estar garantizado. Los profesores interactivos normalmente facilitan más el aprendizaje que los no interactivos, hacen que los alumnos participen más en las clases y alteran el grado de motivación en el aula.

La interacción juega un papel fundamental en el nuevo modelo de enseñanza-aprendizaje en el que el alumno se convierte en el centro del aprendizaje por tener que desarrollar sus destrezas comunicativas, cosa que no es posible si el profesor adopta el papel de controlador o manager. Por el contrario, si el docente de idiomas asume el papel de facilitador del aprendizaje, contribuye a que los alumnos sean más independientes y a la vez más responsables de su propio aprendizaje.

Aprender una lengua es un proceso dinámico y creativo. De ahí que, como profesores preocupados por mejorar nuestra práctica docente, hemos de procurar crear situaciones auténticas dentro del aula, de modo que se refleje el uso de la lengua que encontramos en contextos reales de comunicación. Por esta razón, los profesores con una vocación interactiva debemos modificar la tendencia a monopolizar el discurso para que el alumno asuma un papel activo en el aula de modo que en las clases predominen la cooperación y la interacción.

Una de las ideas principales de este artículo es que el aprendizaje de una lengua extranjera se facilita si el alumno es consciente de los diferentes papeles que puede asumir el profesor y si asume que poner énfasis en algunos de dichos papeles (facilitador, motivador, etc.) en detrimento de otros (instructor, organizador, etc.) implica que el alumno ha de asumir más responsabilidad en el proceso de enseñanza-aprendizaje. Potenciar que el aprendizaje esté centrado en el alumno implica un cambio en el papel del profesor y una adecuación de las metodologías docentes al aprendizaje presencial y no presencial en la que la tutoría juega un papel fundamental, así como una organización de la docencia en la que se presta especial atención al tiempo y al esfuerzo que el alumno emplea, de modo que se fomente el aprendizaje autónomo. 


\section{RefERENCIAS}

Alcaraz E. y B. Moody (1983). Didáctica del inglés: metodología y programación. Madrid: Alhambra.

Alcón Soler, E. (2001). "Interacción y aprendizaje de segundas lenguas en el contexto institucional del aula”, en Pastor Cesteros, S. y V. Salazar García (eds.) Tendencias y líneas de investigación en adquisición de segundas lenguas. Estudios de Lingüística. Alicante, 271-287.

Aleson Carbonell, M., J. Bueno Alonso, V. Domínguez Lucena, M. García Seppere, L. Gómez García, C. Marimón Llorca, T. Morell Moll, C. Puche López, F. Ramos López, C. Segura Llopes (2005) "Perfiles profesionales y competencias para las filologías", en Frau Llinares M.J. y N. Sauleda Parés (eds.) Investigar en diseño curricular. Redes de docencia en el Espacio Europeo de Educación Superior. . Vol. II. Alcoy: Marfil, 125-155.

Carrasco Embuena, V. y C. Lapeña Pérez (2005). "La acción tutorial en la Universidad de Alicante", en Frau Llinares M.J. y N. Sauleda Parés (eds.) Investigar en diseño curricular. Redes de docencia en el Espacio Europeo de Educación Superior. Vol. II. Alcoy: Marfil, 329-358.

Carriscondo Esquivel, F. (2002) Proyecto Docente. Málaga: Universidad de Málaga.

Christie, F. (2000). "The language of Classroom Interaction and Learning”, en Unsworth, L. (ed.) Researching Language in Schools and Communities. Functional Linguistics Perspectives. London: Cassell, 184-244.

Cohen, A.D. (1998). Strategies in Learning and Using a Second Language. London: Longman.

Consolo, D.A. (2000). "Teachers' Action and Student Oral Participation in Classroom Interactio, en Hall, J.K. y L.S. Verplaetse (eds.) Second and Foreign Language Learning Through Classroom Interaction. Mahwah: Lawrence Erlbaum Associates, 91-107.

Council of Europe (2001). Common European Framework of Reference for Languages: Learning, Teaching and Assessment. Cambridge: Cambridge University Press. (ver http: //www.coe.int)

De Sylva Joyce, H. y A. Burns (1999). Focus on Grammar. Sydney: NCELTR.

Ellis, R. (1990). Instructed Second Language Acquisition. Learning in the Classroom. Oxford: Blackwell.

Ellis, R. (1994). The Study of Second Language Acquisition. Oxford: Oxford University Press.

Ellis, R. (1997). Second Language Acquisition. Oxford: Oxford University Press.

González J y R. Wagenaar (2003). Tuning Educational Structures in Europe. Informe final. Proyecto Piloto-Fase 1. Bilbao: Universidad de Deusto.

Hall, J.K. (2000). "Classroom Interaction and Additional Language Learning: Implications for Teaching and Research", en Hall, J.K. y L. Stoops Verplaetse (eds.) Second and Foreign Language Learning Through Classroom Interaction. Mahwah: Lawrence Erlbaum Associates, 287-298.

Hall, J.K. y L.S. Verplaetse (2000) "Second and Foreign Language Learning Through Classroom Interaction", en Hall, J.K. y L.S. Verplaetse (eds.) Second and Foreign Language Learning Through Classroom Interaction. Mahwah: Lawrence Erlbaum Associates, 1-20.

Harmer, J. (2001). The Practice of English Language Teaching. Tercera edición. Londres/Nueva York: Longman.

Lasnier, F. (2000). Réussir la formation par compétences. Montréal: Guérin.

Legutke M. y H. Thomas (1991). Process and Experience in the Language Classroom. Londres/ Nueva York: Longman.

Martínez Lirola, M. (2005). "Systemic functional analysis of teacher's discourse and his/her influence in the learning process". Actas del I Congreso de Internacional de Análisis del Discurso. Valencia: Servicio de Publicaciones de la Universidad de Valencia, 1-9.

Mateo Martínez, J. (1999). La enseñanza universitaria de las lenguas extranjeras. Alicante: Servicio de Publicaciones de la Universidad. 
McDonough, J. y C. Shaw (1993). Materials and Methods in ELT. Oxford: Blackwell.

Morell Moll, T. (2004) . La interacción en la clase magistral. Alicante: Servicio de Publicaciones de la Universidad.

Perrenoud, P. (1999) Dix nouvelles competénces pour enseigner. Paris: ESF editor.

Stern, H.H. (1992). Issues and Options in Language Teaching. Oxford: Oxford University Press. Tsui, A.B.M. (2001). "Classroom Interaction”, en R. Carter y D. Nunan (eds.) The Cambridge Guide to Teaching English to Speakers of Other Languages. Cambridge: Cambridge University Press, 120-125.

Vez, J.M. (2001). Formación en Didáctica de las Lenguas Extranjeras. Rosario: Homo Sapiens. 